\title{
Commodity-Based 2PC for Arithmetic Circuits ${ }^{\star}$
}

\author{
Ivan Damgård, Helene Haagh, Michael Nielsen, and Claudio Orlandi \\ Department of Computer Science, DIGIT, Aarhus University \\ $\{$ ivan, orlandi\}@cs.au.dk
}

\begin{abstract}
We revisit the framework of Commodity-based Cryptography presented by Beaver (STOC'97) with a focus on updating the framework to fit with modern multiparty computation (MPC) protocols. We study the possibility of replacing the well-known preprocessing model with a commodity-based setting, where a set of independent servers (some of which may be corrupt) provide clients with correlated randomness. From this, the clients then distill correct and secure correlated randomness that they can use during the online phase of the MPC protocol. Beaver showed how to do OT with semi-honest security in the commodity setting. We improve on Beaver's result as follows: In a model where one of two clients and a constant fraction of the servers may be maliciously corrupted, we obtain unconditionally secure multiplication triples and oblivious linear evaluations (OLEs) such that the amortized communication cost of one triple/OLE is a constant number of field elements (when the field is sufficiently large). We also report on results from an implementation of the OLE protocol. Finally, we suggest an approach to practical realization of a commodity based system where servers need no memory and can be accessed asynchronously by clients, but still a maliciously corrupt client cannot get data he should not have access to.
\end{abstract}

Keywords. Secure Two-Party Computation, Information Theoretic Security, Oblivious Linear Evaluation, Commodity-based Cryptography.

\section{Introduction}

In commodity-based cryptography as defined in Bea97, we have a set of clients (typically 2) and a set of servers. The clients want to use the servers to help them implement some cryptographic primitive in a way that is faster or more secure than if the clients were on their own - even if some of the servers and clients are corrupted. The primitive we focus on here is secure function evaluation, or primitives that are complete for this purpose, such as creation of random Beaver triples or oblivious linear evaluations (OLEs) (see more details on these below).

While the client-server model has been used multiple times to improve performances of MPC protocols (e.g. BLW08 $\mathrm{BCD}^{+} 09$ JNO14), the commodity-based

\footnotetext{
* Research funded by: the European Research Council (ERC) under the European Unions's Horizon 2020 research and innovation programme under grant agreement No 669255 (MPCPRO), No 803096 (SPEC) and No 731583 (SODA); the Danish Independent Research Council under Grant-ID DFF-6108-00169 (FoCC);
} 
model is significantly different. In particular, what sets the commodity-based model apart from other client-server models are the requirements made on the communication between servers and clients (we recall the formal definition in Appendix B.2):

- Each server should be oblivious to the existence, identities and number of other servers, so no communication takes place between servers.

- The interaction between client $i$ and server $j$ should take the form of a 2message protocol where the client sends a request $q_{i, j}$ and the server returns a response $r_{i, j}$.

- $q_{i, j}$ should be independent of the client's input (apart from its length) and of any previous communications with the servers.

The idea behind the commodity-based model is that it should be easy for a server to set up a business where it provides resources to anyone who is willing to pay, and on the other hand clients can decide to access as many servers as they see fit in order to gain confidence that at least some fraction of the material received is securely and correctly generated.

In Bea97 it was shown how to do 2-party OT and hence 2-party secure computation in the commodity model, assuming only passive corruption of one client and a minority of the servers ${ }^{1}$ Note that in Bea97] it is also claimed that the protocol can be modified to tolerate active corruptions, but no full protocol description nor security proof is provided. Since then, no work seems to have appeared in the commodity model ${ }^{2}$ Recently, in [ST19, Smart et al. present a protocol for secure computation by multiple clients that is "almost" in the commodity model, in the sense that the servers need to interact prior to serving the clients, but not during the actual protocol.

In this paper, we revisit the commodity-based model and improve on Beaver's original result in several ways.

We present two protocols: one that produces batches of OLEs for two clients over any field. The protocol has statistical security against a bounded number of maliciously corrupted servers and 1 maliciously corrupted client. This improves on Beaver's result since: we can deal with arbitrary fields, the mechanism for dealing with active security is more efficient and (crucially) proven secure and, we modify the protocol so that it allows to produce batches of OLEs at the price of tolerating fewer corruptions, thus allowing for meaningful security-efficiency tradeoff. Our second protocol produces multiplication triples over any field for two clients with statistical security against a bounded number of maliciously corrupted servers or 1 maliciously corrupted client. While the security guarantees of this protocol are weaker than the first one, the protocol is more efficient

1 This corruption bound is clearly optimal for information theoretic security: if one of the two clients and half the servers could be corrupt, then we would immediately get a 2-party information theoretically secure OT which is well known to be impossible.

${ }^{2}$ In 2015 , Tonicelli et al. $\mathrm{TND}^{+} 15$ proposed a protocol which they claim to be in the commodity-based model. However, their construction assumes that the servers are trusted, as opposed to our constructions and the original paper by Beaver. 
and therefore it could provide an interesting security-efficiency tradeoffs in some application. In Appendix A we illustrate how our constructions fit in the bigger picture of performing secure 2-party computation. Furthermore, we suggest an approach to practical realization of a commodity-based system where servers can be fully stateless and can be accessed asynchronously by clients.

Constructing Commodity-based OLEs. Our first contribution (in Section 2) is a novel protocol that produces batches of $m$ OLE $\$ 3$ over any field for two clients with statistical security against $t$ maliciously corrupted servers and one client assuming that there are $n=2 t+2 m+1$ servers. This protocol works in the commitment hybrid model. We also show how to do without commitments if we assume $n=2 t+2 m+3$ servers. Thus, we can achieve essentially the optimal corruption threshold by setting the batch size to be $m=1$, while if one is willing to assume a larger number of honest players it is possible to achieve higher amortized efficiency. When the underlying field is large (of size exponential in the security parameter), our protocol requires each server to supply just one OLE to the clients, and sending a constant number of field elements between the clients. This means that we can set both $t$ and $m$ to be $\Theta(n)$ and obtain an amortized communication cost per OLE corresponding to a constant number of field elements while still tolerating a constant fraction of corrupted servers. The computational work is dominated by polynomial interpolation, so by standard FFT techniques the computational complexity for $m \in \Theta(n)$ OLEs is $n \cdot \operatorname{polylog}(n)$ elementary field operations.

We emphasize that the protocol between the clients and each individual server is independent of $n, t$ and $m$. Thus the clients can collect data from any number of servers and later decide on the fly how many OLE's they think it will be secure to extract. Concretely, the clients may agree on number of servers $n$ and corruption threshold $t$ which determine the maximum $m$ by our main theorem. Now, to obtain a total of $M$ OLEs, the clients run $l=M / m$ parallel instances of the whole protocol, which is secure by parallel UC composition.

Although the goal of our protocol is OLE, we construct an equivalent but randomized version, called ROLE. This randomized primitive outputs to the clients random $a, b$ as well as $c_{A}$ and $c_{B}$ where $a b=c_{A}+c_{B}$ (see Appendix B.4). This is sufficient for 2-party computation with good concrete efficiency: ROLE trivially implies passively secure multiplication. But we can also use the ROLEs to produce authenticated multiplication triples allowing us to achieve malicious (and information theoretic) security.

In $\mathrm{DGN}^{+} 17$, it was shown that actively secure 2-party computation can be built from actively secure OLE at an amortized price of 22 OLEs per multiplication. It was recently announced that this has been now improved to $14^{4}$ Using our protocol, this translates to asking for 14 ROLEs from each server per secure multiplication (Given our ROLE protocol, it is also possible to implement secure

\footnotetext{
3 The OLE (Oblivious Linear Evaluation) primitive is defined as follows: Alice inputs values $a$ and $b$ in some field $\mathbb{F}$ and Bob inputs $x \in \mathbb{F}$. Alice learns nothing and Bob learns $y=a x+b$.

${ }^{4}$ Personal communication.
} 
computation for more than two parties, by having each pair of parties constructing the necessary number of ROLEs with the help of the commodity servers). The construction from $\mathrm{DGN}^{+} 17$, works by building authenticated multiplication triples from OLE. Such a triple consists of random values $x, y, z$ with $x y=z$ as well as additive sharings among Alice and Bob of the three values. Moreover, the shares are authenticated using an unconditionally secure MAC scheme to prevent cheating.

In more details, our protocol for ROLE works as follows: the clients ask each server for a ROLE $\left(a_{i}, b_{i}, c_{A, i}, c_{B, i}\right)$, where Alice holds $\left(a_{i}, c_{A, i}\right)$ and Bob holds $\left(b_{i}, c_{B, i}\right)$. Alice and Bob then create two polynomials $A(X)$ and $B(X)$ of degree $d$ by using some of the $a_{i}$ 's and $b_{i}$ 's. Then they jointly create two polynomials $C_{A}(X)$ and $C_{B}(X)$ of degree $2 d$ by using some of the $c_{A, i}$ 's and $c_{B, i}$ 's, and to get the rest of the points they use the remaining unused ROLEs from the servers to multiply $A(X)$ and $B(X)$. If every party behaved honestly, then Alice and Bob will now hold four polynomials that satisfy the following equation:

$$
A(x) \cdot B(x)=C_{A}(x)+C_{B}(x) \quad \text { for all } x \in \mathbb{F} .
$$

However, if one of the servers provided a bad ROLE or one of the clients behaved inconsistently during the protocol, then some of the polynomials might not be well defined, and hence the input/output is not be well defined either. To be able to detect cheating, we introduce a check phase, where each client chooses a random challenge (a random field element), where the other client must prove that the equation holds when evaluating the polynomials on the challenge value. The non-trivial part of our proof is to show that just one such check in each direction is sufficient to ensure that well-defined polynomials exist for a corrupt client (whenever the protocol does not abort). If the protocol does not abort, then the clients can output a valid ROLE by computing $A(\sigma) \cdot B(\sigma)=C_{A}(\sigma)+C_{B}(\sigma)$ where $\sigma$ is a predefined and unused field element. The check for dealing with active adversaries, as well as its analysis, are the main changes we introduce to the original protocol by Beaver, which was only proved to be passive secure.

Implementation of Commodity-based OLEs. We experimentally validate our OLE protocol in Section 3 by implementing it and testing it on Amazon Cloud. The servers were spread over different locations ( 5 different continents). We give detailed timings for different settings of parameters. As an example, when we want to tolerate 5 corrupt servers and hence involve 13 servers in total, the latency for generating an OLE is about $1 \mathrm{msec}$. The amortized time for one OLE ranges from $0.02 \mathrm{msec}$ to $0.5 \mathrm{msec}$ when the field size in bits ranges from 32 to 2048. Note that a growth in wall clock time when the field size grows is to be expected: although the number of field elements to send is constant in the field size, we need to send and process more bits when the field size is larger. These timings are for the case where each instance of the protocol runs with $m=1$, so we can increase $m$ and decrease $t$ and get better amortized times. For instance, if we are willing to assume at most 1 corrupted server out of 13 , one instance of the same protocol can produce batches of 5 OLEs at the time and the amortized times instead vary between 0.004 and $0.1 \mathrm{msec}$. 
With the known reductions from multiplication triples to OLE, our performance numbers show that we can preprocess a secure multiplication triple in amortized time between 0.08 and $0.4 \mathrm{msec}$ for a field size of $128 \mathrm{bits}$, depending on the assumed corruption threshold. For a very rough comparison, the recent "Overdrive" protocol [KPR18, which does the same task using only communication between the two clients, takes roughly $0.03 \mathrm{msec}$ per multiplication in a LAN setting. We emphasize that it does not make sense to compare the timings directly because the hardware set-ups are different and the achieved types of security are different: computational security in the "Overdrive" protocol versus unconditional security assuming an honest server majority in our protocol. Nevertheless, we believe the numbers show that our protocol is indeed applicable in a practical setting.

Constructing Commodity-based Multiplication Triples. As an additional contribution (in the full version [DHNO19]) we present a novel protocol for constructing multiplication triples directly (instead of producing OLE first and then using known reductions). For malicious (and information theoretic) security the standard goal is to produce authenticated multiplication triples. So it may seem natural to ask the servers for such triples and try to extract a secure triple from what we get. However, this does not work: the authentication in a triple involves unconditionally secure MACs and a server will of course know the MAC keys involved in its own triples. If this server as well as, say, Alice is corrupt, then Alice can cheat with those MACs. This problem can be solved in a weaker corruption model where either some servers or a client can be corrupt (but not both).

The high-level idea of the triple protocol closely follows the structure of the OLE protocol with some variation. The clients obtain random triples authenticated under different global keys from the servers, which means that the triples cannot be combined. To solve this, we observe that the MACs are keyhomomorphic, which allows the clients to adjust the global MAC key with one round of interaction. Once the MACs are adjusted to use the same key, the clients can combine the triples in a similar manner as the OLE protocol.

Allowing Servers to be Memoryless. As a final contribution, we suggest an approach for the practical realization of a commodity based system. For the sake of presentation, we assume in most of the paper that there are secure channels between each pair of client and server. Also, we allow servers to remember which clients they talked to and what was sent (so that output to different clients can be properly correlated). Those assumptions are inconvenient in practice and therefore, in Appendix $\mathrm{D}$, we show how to get rid of both requirements. It will clearly be an advantage if the servers do not need memory in the sense that they do not have to remember who they talked to or what was sent. This would mean that a server will not have to administrate identities and log-in credentials, and could simply generate the required data on the fly, say, against payment in some cryptocurrency. 
An obvious problem with this goal is that the data sent to two clients Alice and Bob must be correlated and a memoryless server cannot make this happen on its own. We solve this by letting Alice and Bob interact before talking to the severs, in order to choose a common nonce $n_{A B}$. Then Alice can send ("Alice", $n_{A B}$ ) to the server who will use this and a private PRF key to generate Alice's data. We can do something similar for Bob. However, this is not secure: if Alice is corrupt, she can send both ("Alice", $n_{A B}$ ) and ("Bob", $n_{A B}$ ) to each honest server, get Bob's data and break the protocol. We show how this can be solved by generating the nonce such that Alice and Bob each know different secrets relating to the nonce and hence cannot impersonate the other party towards the server.

Alternatives to the Commodity Model. The commodity model has not received much attention since its introduction, but a very large amount of work has been done on secure computation in general. So we should of course ask ourselves, if some of this work has made the model redundant. Now, if two clients want to do secure computation, two obvious alternatives are well known that allow the clients to do it themselves: first, one could use garbled circuits. This will be constant round, but introduces an overhead on communication that depends on security parameter and also grows with the size of the underlying field (if the goal is arithmetic computation). A second alternative is to do GMW style arithmetic computation using authenticated multiplication triples that the clients generate themselves. If the underlying field is not too large, the triples can be built very efficiently using OT extension KOS16, but we get an overhead that grows linearly with the bit-size of a field element. Another recent approach generates OLEs from noisy encoding-style assumptions with constant overhead $\mathrm{ADI}^{+} 17$, but only with passive security. For specific applications that use scalar-vector multiplication with long vectors, one may consider the generation of vector- $\mathrm{OLE}^{5}$ as in $\mathrm{ADI}^{+} 17$ obtaining rate $1 / 3$. This primitive was recently improved to rate $1 / 2$ using compression [BCGI18, that utilize function secret-sharing to enable a small "sparse" vector-OLE to be locally expanded to larger width.

Since it is clear that any solution involving only the two clients must be based on some intractability assumption, such solutions are incomparable to the commodity model with respect to security: we are replacing trust in a computational assumption by trust in some fraction of the servers. But even if we ignore this issue, what we can do in the commodity model seems to be competitive because our protocol has active security and the overhead of doing a secure multiplication is constant as the field size grows. We do not know any solution with these properties that the clients could execute themselves.

The commodity model is somewhat related to the idea of combiners: for instance, an Oblivious Transfer (OT) combiner HIKN08 $\mathrm{HKN}^{+} 05$ is a protocol that gets (black-box) access to a number of OT implementations, some of which may be faulty, and the goal is now is to build a secure OT. One may think of

\footnotetext{
${ }^{5}$ In the width- $w$ vector-OLE primitive, Alice inputs $\boldsymbol{a}, \boldsymbol{b} \in \mathbb{F}^{w}$ and Bob $x \in \mathbb{F}$. Alice learns nothing and Bob learns $\boldsymbol{y}=\boldsymbol{a} x+\boldsymbol{b}$
} 
the given OT implementations as corresponding to the servers in the commodity model. However, the models are incomparable: on one hand, the combiner model is more restrictive since its "servers" are assumed to only implement a certain primitive. On the other hand, a combiner may make many correlated calls to the "servers".

\section{Commodity-based Oblivious Linear Evaluation}

In this section, we present a commodity-based protocol for ROLE, which will combine commodities in the form of ROLE's from $n$ servers into a secure ROLE (see Appendix B.4 for the formal definition). We consider the setting where one client and up to $t$ of the servers are maliciously corrupt. The protocol is presented in the commitment-hybrid model. In Appendix C, we show as a corollary a protocol that does not use commitments, at the expense of slightly worse parameters. In the appendix, we furthermore discuss the concrete efficiency of the protocol and how to deal with smaller fields.

The high-level idea of the protocol is to first obtain the ROLE commodities from each server. The clients will use $d+1$ of these to fully define two degree $d$ polynomials $A(X)$ and $B(X)$ held by each party respectively and define points on two degree $2 d$ polynomials $C_{1}(X)$ and $C_{2}(X)$, which shall form a secret sharing of $A(X) \cdot B(X)$. The clients will use the remaining ROLEs to securely compute $A(X) \cdot B(X)$ on agreed upon points until we have obtained enough points to fully define $C_{1}(X)$ and $C_{2}(X)$. After constructing these polynomials, the clients perform a check that with high probability reveals whether the polynomials are valid, that is the equation $A(\gamma) \cdot B(\gamma)=C_{1}(\gamma)+C_{2}(\gamma)$ holds for all $\gamma \in \mathbb{F}$. The resulting ROLEs of the protocol will be defined by a fixed set of points $\sigma_{1}, \ldots, \sigma_{m} \in \mathbb{F}$ on these polynomials:

$$
\begin{aligned}
\boldsymbol{u} & =\left(A\left(\sigma_{1}\right), \ldots, A\left(\sigma_{m}\right)\right) & \boldsymbol{v} & =\left(B\left(\sigma_{1}\right), \ldots, B\left(\sigma_{m}\right)\right) \\
\boldsymbol{w}_{1} & =\left(C_{1}\left(\sigma_{1}\right), \ldots, C_{1}\left(\sigma_{m}\right)\right) & \boldsymbol{w}_{2} & =\left(C_{2}\left(\sigma_{1}\right), \ldots, C_{2}\left(\sigma_{m}\right)\right)
\end{aligned}
$$

Here, one client will output $\boldsymbol{u}, \boldsymbol{w}_{1} \in \mathbb{F}^{m}$ and the other $\boldsymbol{v}, \boldsymbol{w}_{2} \in \mathbb{F}^{m}$ such that $\boldsymbol{u} * \boldsymbol{v}=\boldsymbol{w}_{1}+\boldsymbol{w}_{2}$. The complete protocol $\pi_{\mathrm{ROLE}}$ is presented in Protocol[1.

Theorem 1. Assume that $n=2 t+2 m+1$ and that $|\mathbb{F}|$ is exponential in the security parameter. Then protocol $\pi_{\mathrm{ROLE}}^{m}$ is an implementation of $\mathcal{F}_{\mathrm{ROLE}}^{m}$ in the $\mathcal{F}_{\text {Соммгт }}$-hybrid model with statistical UC-security. The protocol tolerates a static adversary corrupting one client and at most $t \leq \frac{n-2 m-1}{2}$ servers. The simulation is perfect unless an error event occurs, which has probability at most $\frac{n}{|\mathbb{F}|-(n+m)}$.

\section{Protocol $\pi_{\mathrm{ROLE}}^{m}$}

Public: Let $d=t+m$ and $n=2 d+1$ and let $\mathcal{U}=\left\{\gamma_{1}, \ldots, \gamma_{n}\right\}, \mathcal{V}=\left\{\sigma_{1}, \ldots, \sigma_{m}\right\}$ be sets of publicly known distinct points in $\mathbb{F}$. Let $\left\{\mathrm{S}_{1}, \ldots, \mathrm{S}_{n}\right\}$ be the servers and $\{$ Alice, Bob $\}$ the clients. 
Output: Alice gets $\boldsymbol{u}, \boldsymbol{w}_{1} \in \mathbb{F}^{m}$ and Bob get $\boldsymbol{v}, \boldsymbol{w}_{2} \in \mathbb{F}^{m}$ such that $\boldsymbol{u} * \boldsymbol{v}=$ $\boldsymbol{w}_{1}+\boldsymbol{w}_{2}$.

\section{Protocol:}

Stateless oblivious RPC:

1. Alice and Bob: send a request to each server $\mathbf{S}_{i}$ for $i \in[n]$;

2. Server $\mathrm{S}_{i}$ for $i \in[n]$ :

- creates a ROLE $\left(a_{i}, b_{i}, c_{1, i}, c_{2, i}\right) \stackrel{\$}{\leftarrow} \mathbb{F}^{4}$ such that $a_{i} \cdot b_{i}=c_{1, i}+c_{2, i}$;

- sends $\left(a_{i}, c_{1, i}\right)$ to Alice and sends $\left(b_{i}, c_{2, i}\right)$ to Bob;

Computation phase:

3. Alice:

- interpolates polynomial $A$ of degree $d$ by setting $A\left(\gamma_{i}\right)=a_{i}$ for $i \in[d+1]$;

- prepares polynomial $C_{1}$ of degree $2 d$ by setting $C_{1}\left(\gamma_{i}\right)=c_{1, i}$ for $i \in[d+1]$;

4. Bob:

- interpolates polynomial $B$ of degree $d$ by setting $B\left(\gamma_{i}\right)=b_{i}$ for $i \in[d+1]$;

- prepares polynomial $C_{2}$ of degree $2 d$ by setting $C_{2}\left(\gamma_{i}\right)=c_{2, i}$ for $i \in[d+1]$;

5. For $i=d+2, \ldots, n$

a. Alice: sends $k_{i}=A\left(\gamma_{i}\right)-a_{i}$ to Bob;

b. Bob:

- sends $\ell_{i}=B\left(\gamma_{i}\right)-b_{i}$ to Alice;

- sets $C_{2}\left(\gamma_{i}\right)=c_{2, i}+B\left(\gamma_{i}\right) \cdot k_{i}$;

c. Alice: sets $C_{1}\left(\gamma_{i}\right)=c_{1, i}+A\left(\gamma_{i}\right) \cdot \ell_{i}-k_{i} \cdot \ell_{i}$;

6. Alice: interpolates polynomial $C_{1}$ by the $2 d+1$ defined points;

7. Bob: interpolates polynomial $C_{2}$ by the $2 d+1$ defined points;

Check phase:

8. Alice: draw $t_{A} \stackrel{\$}{\leftarrow} \mathbb{F} \backslash(\mathcal{U} \cup \mathcal{V})$ uniformly at random and send $t_{A}$ to Bob;

9. Bob:

- draw $t_{B} \stackrel{\$}{\leftarrow} \mathbb{F} \backslash(\mathcal{U} \cup \mathcal{V})$ uniformly at random;

- Execute Commit $\left(B\left(t_{A}\right), C_{2}\left(t_{A}\right)\right)$ and send $t_{B}$ to Alice;

10. Alice: once commitment has been done, send $\left(A\left(t_{B}\right), C_{1}\left(t_{B}\right)\right)$ to Bob;

11. Bob:

- check if $A\left(t_{B}\right) \cdot B\left(t_{B}\right)=C_{1}\left(t_{B}\right)+C_{2}\left(t_{B}\right)$ and abort if not;

- execute $\operatorname{OPEN}\left(B\left(t_{A}\right), C_{2}\left(t_{A}\right)\right)$;

12. Alice:

- receive $\left(B\left(t_{A}\right), C_{2}\left(t_{A}\right)\right)$ from the opening of the commitment, abort if nothing is received;

- check if $A\left(t_{A}\right) \cdot B\left(t_{A}\right)=C_{1}\left(t_{A}\right)+C_{2}\left(t_{A}\right)$ and abort if not;

Output phase:

13. Alice: output $\boldsymbol{u}=\left(A\left(\sigma_{1}\right), \ldots, A\left(\sigma_{m}\right)\right)$ and $\boldsymbol{w}_{1}=\left(C_{1}\left(\sigma_{1}\right), \ldots, C_{1}\left(\sigma_{m}\right)\right)$;

14. Bob: output $\boldsymbol{v}=\left(B\left(\sigma_{1}\right), \ldots, B\left(\sigma_{m}\right)\right)$ and $\boldsymbol{w}_{2}=\left(C_{2}\left(\sigma_{1}\right), \ldots, C_{2}\left(\sigma_{m}\right)\right)$.

Protocol 1: Protocol for commodity-based ROLE

Proof. The environment corrupts one client and a subset of the servers $\mathcal{C} \subset[n]$ with $|\mathcal{C}| \leq t$. Thus, the environment learns at most $t$ points on all polynomials, and one extra from the check phase. After seeing these points, the environment 
still cannot distinguish whether it is interacting with the ideal world or the real world - that is, the output of the computation (which is $m$ extra points on the polynomial) is compatible with the points that it already received from the simulator (which does not learn the output of the honest party). In other words, a necessary condition for being able to prove unconditional security is to set the degree to $d=t+m$ (since a random such polynomial has $t+m+1$ random coefficients). The protocol uses $2 d+1$ ROLEs in total and thus we need the number of servers to be at least $n \geq 2 d+1=2 t+2 m+1$. In other words, we have that $t \leq \frac{n-2 m-1}{2}$.

The protocol is essentially symmetric, i.e. the only difference is that in the proof for corrupt Bob we need to exploit that Bob has to commit to his answer to Alice's challenge before he sees $A\left(t_{B}\right)$, and hence a corrupt Bob also knows only $t$ points of Alice's polynomial. Thus, in the following we prove security against corrupt Alice.

Let $\mathcal{C} \subset[n]$ denote the set of corrupt servers and $\mathcal{H} \subseteq[n]$ the set of honest servers. For these sets it hold that $|\mathcal{C}| \leq t, \mathcal{C} \cup \mathcal{H}=[n]$, and $\mathcal{C} \cap \mathcal{H}=0$. Note that the servers play two different roles in the protocol: the first $d+1$ servers are used to define the polynomials $A(x)$ and $B(x)$, and the last $n-d-1$ servers are used to compute the remaining points on $C_{1}(x)$ and $C_{2}(x)$.

We start by presenting some facts about the protocol.

Definition 1. For an honest server $i \in \mathcal{H}$, Alice gets $a_{i}$ from the server and (if $i \geq d+2$ ) sends a value $k_{i}$ to Bob. Note that if Alice follows the protocol, then the values $\left\{a_{i}\right\}_{i \in \mathcal{H}, i \leq d+1}$ and $\left\{k_{i}+a_{i}\right\}_{i \in \mathcal{H}, i \geq d+2}$ are all consistent with a polynomial of degree at most d (namely $A(x)$ if Alice was honest). We say that Alice behaves consistently if such a polynomial $A^{*}(x)$ of degree at most $d$ exists.

Lemma 1. If Alice is corrupt, but behaves consistently, then from her interaction with honest servers and Bob, one can compute a uniquely defined view for Alice and all corrupt servers that is consistent with them having followed the protocol up to (but not including) the check phase. This includes polynomials $A^{*}(x), C_{1}^{*}(x)$ of degree at most $d$ and $2 d$ respectively, where if Alice is honest we have $A^{*}(x)=A(x)$ and $C_{1}^{*}(x)=C_{1}(x)$.

Proof. Since Alice behaves consistently this uniquely defines a polynomial $A^{*}(x)$ of degree at most $d$, by the above definition. Now we define a view $\left(a_{i}, b_{i}, c_{1, i}, c_{2, i}\right)$ for each corrupt server $i \in \mathcal{C}$ : The server sent $b_{i}, c_{2, i}$ to honest Bob, so these are fixed. Then if $1 \leq i \leq d+1$, there is no interaction between Alice and Bob so we set $a_{i}=A^{*}\left(\gamma_{i}\right)$ and $c_{1, i}=a_{i} b_{i}-c_{2-i}$. If $d+2 \leq i \leq n$, Alice sent $k_{i}$ and received $\ell_{i}$ from Bob. So we set $a_{i}=A^{*}\left(\gamma_{i}\right)-k_{i}$, and $c_{1, i}=a_{i} b_{i}-c_{2-i}$. This gives a view for each corrupt server which is consistent with $A^{*}(x)$ and honest behaviour. Each honest server $i$, has sent $a_{i}, c_{1, i}$ to Alice and (if $i \geq d+2$ ) she has received a value $\ell_{i}$ from Bob and has sent $k_{i}$ to Bob.

In particular, $c_{1, i}$ is now defined for all $i$ and $k_{i}, \ell_{i}$ are defined for $i \geq d+2$. Therefore we can define the polynomial $C_{1}^{*}(x)$ by simply following the specification of the protocol, namely we set $C_{1}^{*}\left(\gamma_{i}\right)=c_{1, i}$ for $1 \leq i \leq d+1$ and 
otherwise $C_{1}^{*}\left(\gamma_{i}\right)=c_{1, i}+A^{*}\left(\gamma_{i}\right) \ell_{i}-k_{i} \ell_{i}$, and finally we interpolate $C_{1}^{*}(x)$ from these values.

Lemma 2. If Alice is corrupt and does not behave consistently, then the protocol aborts in the check phase except with probability at most $\frac{n}{|\mathbb{F}|-(m+n)}$.

Proof. Consider the values $Q=\left\{a_{i}\right\}_{i \in \mathcal{H}, i \leq d+1} \cup\left\{k_{i}+a_{i}\right\}_{i \in \mathcal{H}, i \geq d+2}$ (see Definition 1). Since Alice does not behave consistently these values are not all consistent with a polynomial of degree at most $d$. We nevertheless define a polynomial $A^{\prime}(x)$ by interpolating from the first $d+1$ values in $Q{ }^{6}$ For all the remaining values in $Q$, we define $\delta_{i}$ by

$$
k_{i}+a_{i}=A^{\prime}\left(\gamma_{i}\right)+\delta_{i}
$$

Note that, by assumption, there exists an index $j \in \mathcal{H}$ and $j>d+2$ such that $\delta_{j} \neq 0$. To simplify the notation, let $\mathcal{H}_{1}$ be the first $d+1$ indexes of the honest servers, i.e. those used for defining polynomial $A^{\prime}(x)$, and let $\mathcal{H}_{2}$ be the remaining honest servers.

On the other hand, for the corrupt servers, one can always fix a view that is consistent with $A^{\prime}(x)$ and with the interaction with (honest) Bob, exactly as in the proof of the previous lemma. Thus we assume (for notational convenience) that $a_{i}$ for $i \in \mathcal{C}$ is defined this way such that $A^{\prime}\left(\gamma_{i}\right)=a_{i}$.

Now, from the communication in the computation phase, for each index $i$ the outcome for Alice and Bob consists of two field elements $C_{1}\left(\gamma_{i}\right)$ and $C_{2}\left(\gamma_{i}\right)$ (of course, corrupt Alice does not necessarily store the $C_{1}\left(\gamma_{i}\right)$ 's, all we mean to say is that they can be computed from the adversary's view). Now, from our definition of $A^{\prime}(x)$ and $\delta_{i}$ and the protocol specification, it is easy to see that $C_{1}\left(\gamma_{i}\right)+C_{2}\left(\gamma_{i}\right)=A^{\prime}\left(\gamma_{i}\right) B\left(\gamma_{i}\right)$ if $i \in \mathcal{C} \cup \mathcal{H}_{1}$, and otherwise $C_{1}\left(\gamma_{i}\right)+C_{2}\left(\gamma_{i}\right)=$ $\left(A^{\prime}\left(\gamma_{i}\right)+\delta_{i}\right) B\left(\gamma_{i}\right)=A^{\prime}\left(\gamma_{i}\right) B\left(\gamma_{i}\right)+\delta_{i} B\left(\gamma_{i}\right)$ if $i \in \mathcal{H}_{2}$. For notational convenience, we will define $\delta_{i}=0$ for $i \in \mathcal{C} \cup \mathcal{H}_{1}$, so we have

$$
C_{1}\left(\gamma_{i}\right)+C_{2}\left(\gamma_{i}\right)=A^{\prime}\left(\gamma_{i}\right) B\left(\gamma_{i}\right)+\delta_{i} B\left(\gamma_{i}\right) \text { for } i \in[n]
$$

We can now interpolate polynomials of degree at most $2 d$ from the $C_{1}\left(\gamma_{i}\right)$ 's, the $C_{2}\left(\gamma_{i}\right)$ 's and the $C_{1}\left(\gamma_{i}\right)+C_{2}\left(\gamma_{i}\right)=A^{\prime}\left(\gamma_{i}\right) B\left(\gamma_{i}\right)+\delta_{i} B\left(\gamma_{i}\right)$-values. Because interpolation is linear, this results in polynomials $C_{1}(x), C_{2}(x)$ such that

$$
C_{1}(x)+C_{2}(x)=A^{\prime}(x) B(x)+\Delta(x), \text { where } \Delta\left(\gamma_{i}\right)=B\left(\gamma_{i}\right) \delta_{i} \text { for } i \in[n] .
$$

In the test phase, Bob sends a point $t_{B}$ and Alice returns two field elements, that are "supposed to be" $A^{\prime}\left(t_{B}\right)$ and $C_{1}\left(t_{B}\right)$. We can always write what she actually sends as $\alpha+A^{\prime}\left(t_{B}\right)$ and $\beta+C_{1}\left(t_{B}\right)$, for some $\alpha, \beta$ that the adversary can compute. Bob will check the equation

$$
\left(\beta+C_{1}\left(t_{B}\right)\right)+C_{2}\left(t_{B}\right)=\left(\alpha+A^{\prime}\left(t_{B}\right)\right) B\left(t_{B}\right)
$$

${ }^{6}$ The choice to interpolate from the first $d+1$ values is completely arbitrary, the following argument will work no matter the choice of subset. 
which easily simplifies to $\beta+\Delta\left(t_{B}\right)=\alpha B\left(t_{B}\right)$.

So what we need to argue is that the adversary can guess $\alpha, \beta$ satisfying this equation with only negligible probability. This will turn out to be because he does not have sufficient information about the polynomial $B(x)$. Note that the adversary has seen $t$ values of $B(x)$ from Bob's interaction with the corrupt servers $\left(B\left(t_{A}\right)\right.$ has been committed to but is not revealed yet). Since the degree is $d=t+m$ and $m \geq 1$, the adversary is at least 2 points short of being able to determine $B(x)$. We can therefore assume that the values $B\left(t_{B}\right)$ and $B\left(\gamma_{j}\right)$ are independent and uniform in the view of the adversary, namely $\gamma_{j}$ is the index of an honest server and $t_{B}$ is chosen such that it is never the index of a corrupt server. To emphasize that the values are unknown, we will write $X=B\left(t_{B}\right)$ and $Y=B\left(\gamma_{j}\right)$.

We can imagine giving the adversary extra points so he knows exactly $d-1$ points on $B(x)$, this can only help him. Therefore, using the formulas of interpolation, the adversary can write any value of $B(x)$ as an affine linear combination of $X$ and $Y$ with known coefficients. In particular, this means there exist field elements $\omega_{i}, \eta_{i}, \sigma_{i}$ such that $\Delta\left(\gamma_{i}\right)=B\left(\gamma_{i}\right) \delta_{i}=\omega_{i} X+\eta_{i} Y+\sigma_{i}$. From the values $\omega_{1}, \ldots, \omega_{n}$ we can interpolate exactly one polynomial of degree at most $2 d$, which we call $F(x)$. Likewise we interpolate $G(x)$ from the $\eta_{i}$ 's and $H(x)$ from the $\sigma_{i}$ 's. This immediately implies that

$$
\Delta(x)=F(x) X+G(x) Y+H(x)
$$

The meaning of this equation is that the polynomials $F(x), G(x)$ and $H(x)$ are fixed in the sense that they do not depend on the choice of $B(x)$ (and hence of $X, Y)$. So in the adversary's view, the polynomial $\Delta(x)$ depends linearly on the two (random) values $X, Y$ as described by the equation.

Note that for $i=j$, we have $\delta_{j} B\left(\gamma_{j}\right)=\delta_{j} Y=\omega_{j} X+\eta_{j} Y+\sigma_{j}$ which can only be true for random $X$ and $Y$ if $\omega_{j}=\sigma_{j}=0$ and $\eta_{j}=\delta_{j} \neq 0$. So this implies that $G(x)$ is not the 0 -polynomial. Hence the above equation that the adversary must try to satisfy becomes:

$$
\beta+F\left(t_{B}\right) X+G\left(t_{B}\right) Y+H\left(t_{B}\right)=\alpha X
$$

Which can be rewritten as $\left(F\left(t_{B}\right)-\alpha\right) X+G\left(t_{B}\right) Y+\beta+H\left(t_{B}\right)=0$.

Note that we can think of the experiment done as follows: first we choose $t_{B}$ at random from a set of size $|\mathbb{F}|-(m+n)$, then the adversary chooses $\alpha, \beta$ and then we choose $X, Y$ independently and uniformly at random in $\mathbb{F}$. It is then clear that if $\left(F\left(t_{B}\right)-\alpha\right) \neq 0$ then the left-hand side is uniformly random and so is 0 with probability $1 /|\mathbb{F}|$. On the other hand, if $\left(F\left(t_{B}\right)-\alpha\right)=0$, we can use the fact that $G(x)$ is non-zero and has degree at most $2 d$ to conclude that $G\left(t_{B}\right)=0$ with probability at most $2 d /(|\mathbb{F}|-(m+n))$. But if $G\left(t_{B}\right) \neq 0$, then again the left-hand side is uniformly random and is 0 with probability $1 /|\mathbb{F}|$. We conclude that the equation is satisfied with probability at most

$$
\frac{2 d}{|\mathbb{F}|-(m+n)}+\frac{1}{|\mathbb{F}|} \leq \frac{n}{|\mathbb{F}|-(m+n)}
$$


Having proved the lemmas, we present a simulator $\mathcal{S}_{A}$ (see Simulator 1) which provides statistically indistinguishable simulation of the protocol $\pi_{\mathrm{ROLE}}$ against a malicious adversary that corrupts Alice and $t$ servers. The simulator basically runs its own instance of the protocol with corrupt Alice and servers, playing honestly for Bob and the honest servers. There is, however, an important difference: During the check phase, the simulator aborts under a different condition than in the real protocol: While an honest Bob in the real protocol aborts only if the values sent by Alice do not satisfy the right relation with the polynomials held by Bob, the simulator will also abort if Alice does not behave consistently. Now, there two cases to consider:

1. Alice behaves consistently: in this case, the simulator follows the protocol until the end, so it is clear that simulation of the adversary's view of the protocol is perfect. Furthermore, it follows by Lemma 1 that the simulator extracts the only possible candidate for Alice's output shares, given the interaction with honest players, so what it sends to the functionality is correct. Hence the only difference between the real and the ideal process is that in the real process, Bob's output is extracted from his view of the protocol, whereas in the ideal process it is chosen by the functionality (but consistently with Alice's shares). This makes no difference: Alice has seen $t+1$ points on the polynomial $B(x)$ and since the degree is $d=t+m$ this leaves $m$ degrees of freedom which means that the values $B\left(\sigma_{1}\right), \ldots, B\left(\sigma_{m}\right)$ are random and independent of the adversary/environment's view of the protocol. So in this case, the real and ideal process are perfectly indistinguishable.

2. Alice does not behave consistently: in this case the ideal process always aborts, but by Lemma 2 the real process does the same, except with negligible probability. Thus in this case, the processes are statistically indistinguishable.

Simulator 1: Simulator $\mathcal{S}_{A}$ against corrupt Alice

The simulator starts by initializing copies of the code for the honest servers, for

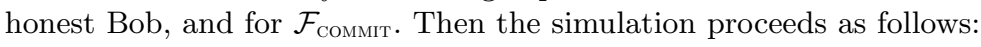

Stateless oblivious RPC and Computation phase:

1. Let the simulator's copies of the honest servers and Bob interact with corrupt Alice and the corrupt servers (which are controlled by the environment).

2. When the computation phase is done, check whether Alice has acted consistently (see Definition 1).

3. If Alice has not acted consistently, set a flag will-abort = true. Else (Alice has acted consistently), do as follows:

(a) Compute polynomials $A^{*}(x), C_{1}^{*}(x)$ as guaranteed by Lemma 1

(b) Compute $\hat{\boldsymbol{u}}=\left(A^{*}\left(\sigma_{1}\right), \ldots, A^{*}\left(\sigma_{m}\right)\right)$. $\hat{\boldsymbol{w}}_{1}=\left(C_{1}^{*}\left(\sigma_{1}\right), \ldots, C_{1}^{*}\left(\sigma_{m}\right)\right)$.

Send (corrupt, $\left.\mathrm{A},\left(\hat{\boldsymbol{u}}, \hat{\boldsymbol{w}}_{1}\right)\right)$ to the ideal functionality $\mathcal{F}_{\mathrm{ROLE}}^{m}$;

Check phase:

1. Let the simulator's copies of Bob and $\mathcal{F}_{\text {сомміт }}$ do the check phase with corrupt

Alice. If the test done by Bob fails, set the flag will-abort = true.

Output phase: 
1. If will-abort $=$ true, abort the protocol.

Else, send (deliver, A) and (deliver, B) to the ideal functionality $\mathcal{F}_{\mathrm{ROLE}}^{m}$.

\section{Implementation}

We implement and measure timings for the commodity-based OLE protocol. ${ }^{7}$ The two clients Alice and Bob are set up on a basic LAN and will connect to some number of servers around the world. Since the experiments are identical up to the output for different tradeoff choices of $t$ and $m$, we implement the setting of generating one OLE $m=1$ with maximum adversarial threshold $t \leq \frac{n-3}{2}$.

Instantiations. We use a basic OpenSSL (version 1.1.0) setup to implement a public key infrastructure for the clients to authenticate servers. Our setting consists of a single root certificate authority, trusted by each client, and who have signed certificates to each server. This setup is easily used in the real world, as two clients can simply agree on some domain name and rely on root certificates already included in the system to do hostname validation.

All finite fields are implemented using GNU Multiple Precision Arithmetic Library and when testing a $b$-bit prime field, we refer to $\mathbb{Z}_{p}$ for the largest $p<2^{b}$. We instantiate the hash function $\mathcal{H}$ as SHA256. For sampling random numbers, we construct a PRG by using the AES instruction set in counter mode. This PRG takes a seed $s$ of arbitrary length and set the AES key to be the 128 first bits of $\mathcal{H}(s)$. To generate a random field element from $\mathbb{Z}_{p}$, we sample $b=\left\lceil\log _{2} p\right\rceil$ random bits from the PRG repeatedly until it represents a valid element.

We fix the polynomial evaluation points $\gamma_{1}, \ldots, \gamma_{n}$ to be $1, \ldots, n$, and set the extraction point $\sigma_{1}=0$. We use the following preprocessed variant of Lagrange interpolation to fas $t^{8}$ evaluate $f(x)$ where $f$ is a degree $d$ polynomial represented by $d+1$ points $y_{1}, \ldots, y_{n}$ and $y_{i}=f(i)$. First preprocess $\delta_{i j}=(i-j)^{-1}$ and $\lambda_{i j}=j \cdot \delta_{i j}$ for $i, j \in[d+1]$, and then compute the point $f(x)$ as:

$$
f(x)=\sum_{i=1}^{d+1} y_{i} \prod_{\substack{j=1 \\ j \neq i}}^{d+1} x \delta_{i j}-\lambda_{i j}
$$

Set-up and results. The two clients are tested on a basic LAN setup consisting of two identical machines each with a i7-3770K CPU running at $3.5 \mathrm{GHz}, 32 \mathrm{~GB}$ of RAM and connected via $1 \mathrm{GbE}$ with a $0.15 \mathrm{~ms}$ delay. The servers are set up on Amazon Cloud using M4.LARge instances with 2 vCPUs and are spread across five continents namely North America (N. Virginia), South America (São Paulo), Europe (Ireland), Asia (Mumbai) and Australia (Sydney). The Internet

\footnotetext{
7 The sources used for the benchmark implementation are available at [source hidden for anonymity]

${ }^{8}$ Fast in practice for low-degree polynomials, but theoretically inferior to the Fast Fourier Transform.
} 
connection for all servers and clients was measured to vary between 200-500 Mbps up and down at the time of testing.

We test the protocol with different field sizes ranging from 32 to 2048 bit and tolerating up to 1,5 or 10 malicious servers colluding with one malicious client. This implies the number of servers used in each setting being 5,13 and 23 respectively. Both clients run a producer-consumer program where the producer is connected to all servers and produce batches of shares from each server to be used for the protocol. The consumer is connected to the other client and consumes a batch by running the protocol in parallel for each OLE to be corrected. All measurements are done as an average over 30 seconds.

First we measure sequential timings for protocol, namely how long time a single consumer (thread) takes to compute a corrected ROLE given the raw material. We test this in two versions, one where the clients are only interested in a single ROLE and another where they want a batch of 1000 . The first version may be interesting in a real-world application where clients wants a single OLE for say a commitment, and serves well as a baseline for OLE protocol comparison. The second version on the contrary shows what to expect, if our protocol is to be used in a subsequent protocol requiring 1000 OLEs. Here, one would expect a batch of 1000 to take 1000 times as long - however the numbers show that this vary between roughly 200 to 900 depending on the field size and number of servers, which is partly due to less network delay. Finally, amortized timings for the protocol are measured. These timings show how many ROLEs we can generate per second. We simply let the machines generate as many ROLEs as possible by turning up the number of consumers. Note that our tests was done on a university network and on shared cloud nodes, which meant inconsistency in available resources. This, together with different parameter choices to maximize parallization (number of threads and batch size), means that we can expect to see jumps in the amortized table, for example for 23 servers and $b=256$ and $b=512$.

\begin{tabular}{|l|c|c|c|}
\hline & $\begin{array}{c}n=5 \\
t=1\end{array}$ & $\begin{array}{c}n=13 \\
t=5\end{array}$ & $\begin{array}{c}n=23 \\
t=10\end{array}$ \\
\hline$b=32$ & $0.301 \mathrm{~ms}$ & $0.460 \mathrm{~ms}$ & $0.930 \mathrm{~ms}$ \\
\hline$b=64$ & $0.317 \mathrm{~ms}$ & $0.465 \mathrm{~ms}$ & $1.162 \mathrm{~ms}$ \\
\hline$b=128$ & $0.333 \mathrm{~ms}$ & $0.803 \mathrm{~ms}$ & $1.294 \mathrm{~ms}$ \\
\hline$b=256$ & $0.980 \mathrm{~ms}$ & $1.388 \mathrm{~ms}$ & $2.311 \mathrm{~ms}$ \\
\hline$b=512$ & $1.372 \mathrm{~ms}$ & $1.891 \mathrm{~ms}$ & $3.301 \mathrm{~ms}$ \\
\hline$b=1024$ & $1.491 \mathrm{~ms}$ & $2.625 \mathrm{~ms}$ & $5.228 \mathrm{~ms}$ \\
\hline$b=2048$ & $1.856 \mathrm{~ms}$ & $4.252 \mathrm{~ms}$ & $9.311 \mathrm{~ms}$ \\
\hline
\end{tabular}

\begin{tabular}{|l|c|c|c|}
\hline & $\begin{array}{c}n=5 \\
t=1\end{array}$ & $\begin{array}{c}n=13 \\
t=5\end{array}$ & $\begin{array}{c}n=23 \\
t=10\end{array}$ \\
\hline$b=32$ & $13.450 \mathrm{~ms}$ & $86.103 \mathrm{~ms}$ & $370.984 \mathrm{~ms}$ \\
\hline$b=64$ & $13.356 \mathrm{~ms}$ & $136.978 \mathrm{~ms}$ & $405.691 \mathrm{~ms}$ \\
\hline$b=128$ & $15.334 \mathrm{~ms}$ & $171.623 \mathrm{~ms}$ & $468.540 \mathrm{~ms}$ \\
\hline$b=256$ & $24.716 \mathrm{~ms}$ & $317.403 \mathrm{~ms}$ & $829.741 \mathrm{~ms}$ \\
\hline$b=512$ & $51.997 \mathrm{~ms}$ & $410.123 \mathrm{~ms}$ & $1792.491 \mathrm{~ms}$ \\
\hline$b=1024$ & $151.008 \mathrm{~ms}$ & $858.411 \mathrm{~ms}$ & $2820.248 \mathrm{~ms}$ \\
\hline$b=2048$ & $371.269 \mathrm{~ms}$ & $2188.804 \mathrm{~ms}$ & $7970.499 \mathrm{~ms}$ \\
\hline
\end{tabular}

Table 1. Sequential timings for one OLE

Table 2. Sequential timings for batch of 1000 OLEs 


\begin{tabular}{|l|c|c|c|}
\hline & $\begin{array}{c}n=5 \\
t=1\end{array}$ & $\begin{array}{c}n=13 \\
t=5\end{array}$ & $\begin{array}{c}n=23 \\
t=10\end{array}$ \\
\hline$b=32$ & $3.570 \mu \mathrm{s}$ & $23.657 \mu \mathrm{s}$ & $89.055 \mu \mathrm{s}$ \\
\hline$b=64$ & $5.801 \mu \mathrm{s}$ & $23.362 \mu \mathrm{s}$ & $105.628 \mu \mathrm{s}$ \\
\hline$b=128$ & $16.867 \mu \mathrm{s}$ & $28.201 \mu \mathrm{s}$ & $106.868 \mu \mathrm{s}$ \\
\hline$b=256$ & $33.101 \mu \mathrm{s}$ & $56.197 \mu \mathrm{s}$ & $191.985 \mu \mathrm{s}$ \\
\hline$b=512$ & $69.297 \mu \mathrm{s}$ & $115.180 \mu \mathrm{s}$ & $2160.664 \mu \mathrm{s}$ \\
\hline$b=1024$ & $118.353 \mu \mathrm{s}$ & $230.487 \mu \mathrm{s}$ & $4938.348 \mu \mathrm{s}$ \\
\hline$b=2048$ & $249.018 \mu \mathrm{s}$ & $516.934 \mu \mathrm{s}$ & $7709.943 \mu \mathrm{s}$ \\
\hline
\end{tabular}

Table 3. Amortized timing for generating one OLE

Using the tradeoff, one can increase $m$ and decrease $t$ to get a protocol with same sequential running time, but with higher throughput i.e. lower amortized timings. For example, the second column for the amortized timings represent $n=13, t=5$ and $m=1$ - but we can get a five time increase in throughput by running the protocol with $n=13, t=1$ and $m=5$. Likewise, for the case of $m=2$, one can decrease $t$ by one to obtain a column with half the amortized timings.

In the case of 128-bit fields, existing protocols providing computational security like the "Overdrive" LowGear protocol KPR18 achieve a secure 128-bit multiplication in roughly 0.03 msec. To compare our protocol roughly, one can set $n=13$ and assume a 14 times overhead by using the optimized ${ }^{9}$ TinyOLE variant, we can compute roughly a secure multiplication between 0.4 and 0.08 msec with a choice of $(t=5, m=1)$ and $(t=1, m=5)$ respectively. We stress the difference between the models used by us and "Overdrive": ours provide unconditional security (assuming $\leq t$ malicious servers colluding) rather than computational security - and we believe these numbers show that our protocol is indeed applicable in a practical setting.

\section{References}

$\mathrm{ADI}^{+}$17. Benny Applebaum, Ivan Damgård, Yuval Ishai, Michael Nielsen, and Lior Zichron. Secure arithmetic computation with constant computational overhead. In CRYPTO (1), volume 10401 of Lecture Notes in Computer Science, pages 223-254. Springer, 2017.

$\mathrm{BCD}^{+}$09. Peter Bogetoft, Dan Lund Christensen, Ivan Damgård, Martin Geisler, Thomas P. Jakobsen, Mikkel Krøigaard, Janus Dam Nielsen, Jesper Buus Nielsen, Kurt Nielsen, Jakob Pagter, Michael I. Schwartzbach, and Tomas Toft. Secure multiparty computation goes live. In Financial Cryptography

\footnotetext{
${ }^{9}$ Which requires 14 OLEs to produce a secure multiplication, by personal communication
} 
and Data Security, 13th International Conference, FC 2009, Accra Beach, Barbados, February 23-26, 2009. Revised Selected Papers, pages 325-343, 2009.

BCGI18. Elette Boyle, Geoffroy Couteau, Niv Gilboa, and Yuval Ishai. Compressing vector OLE. In Proceedings of the 2018 ACM SIGSAC Conference on Computer and Communications Security, CCS 2018, Toronto, ON, Canada, October 15-19, 2018, pages 896-912, 2018.

Bea97. Donald Beaver. Commodity-based cryptography (extended abstract). In Proceedings of the Twenty-Ninth Annual ACM Symposium on the Theory of Computing, El Paso, Texas, USA, May 4-6, 1997, pages 446-455, 1997.

BLW08. Dan Bogdanov, Sven Laur, and Jan Willemson. Sharemind: A framework for fast privacy-preserving computations. In Computer Security - ESORICS 2008, 13th European Symposium on Research in Computer Security, Málaga, Spain, October 6-8, 2008. Proceedings, pages 192-206, 2008.

Can01. Ran Canetti. Universally Composable Security: A New Paradigm for Cryptographic Protocols. In FOCS, pages 136-145, 2001.

$\mathrm{DGN}^{+}$17. Nico Döttling, Satrajit Ghosh, Jesper Buus Nielsen, Tobias Nilges, and Roberto Trifiletti. Tinyole: Efficient actively secure two-party computation from oblivious linear function evaluation. In CCS, pages 2263-2276. ACM, 2017.

DHNO19. Ivan Damgård, Helene Haagh, Michael Nielsen, and Claudio Orlandi. Commodity-based 2pc for arithmetic circuits. Cryptology ePrint Archive, Report 2019/705, 2019. https://eprint.iacr.org/2019/705

HIKN08. Danny Harnik, Yuval Ishai, Eyal Kushilevitz, and Jesper Buus Nielsen. Otcombiners via secure computation. In Theory of Cryptography, Fifth Theory of Cryptography Conference, TCC 2008, New York, USA, March 19-21, 2008., pages 393-411, 2008.

$\mathrm{HKN}^{+}$05. Danny Harnik, Joe Kilian, Moni Naor, Omer Reingold, and Alon Rosen. On robust combiners for oblivious transfer and other primitives. In Advances in Cryptology - EUROCRYPT 2005, 24th Annual International Conference on the Theory and Applications of Cryptographic Techniques, Aarhus, Denmark, May 22-26, 2005, Proceedings, pages 96-113, 2005.

$\mathrm{IKM}^{+}$13. Yuval Ishai, Eyal Kushilevitz, Sigurd Meldgaard, Claudio Orlandi, and Anat Paskin-Cherniavsky. On the power of correlated randomness in secure computation. In Theory of Cryptography - 10th Theory of Cryptography Conference, TCC 2013, Tokyo, Japan, March 3-6, 2013. Proceedings, pages 600620, 2013.

JNO14. Thomas P. Jakobsen, Jesper Buus Nielsen, and Claudio Orlandi. A framework for outsourcing of secure computation. In Proceedings of the 6th edition of the ACM Workshop on Cloud Computing Security, CCSW '14, Scottsdale, Arizona, USA, November 7, 2014, pages 81-92, 2014.

KOS16. Marcel Keller, Emmanuela Orsini, and Peter Scholl. MASCOT: faster malicious arithmetic secure computation with oblivious transfer. In ACM Conference on Computer and Communications Security, pages 830-842. ACM, 2016.

KPR18. Marcel Keller, Valerio Pastro, and Dragos Rotaru. Overdrive: Making SPDZ great again. In EUROCRYPT (3), volume 10822 of Lecture Notes in Computer Science, pages 158-189. Springer, 2018.

NP99. Moni Naor and Benny Pinkas. Oblivious transfer and polynomial evaluation. In Proceedings of the Thirty-First Annual ACM Symposium on Theory of Computing, May 1-4, 1999, Atlanta, Georgia, USA, pages 245-254, 1999. 
Rab05. Michael O. Rabin. How to exchange secrets with oblivious transfer. IACR Cryptology ePrint Archive, 2005:187, 2005.

ST19. Nigel P. Smart and Titouan Tanguy. Taas: Commodity MPC via triples-asa-service. In CCSW@CCS, pages 105-116. ACM, 2019.

$\mathrm{TND}^{+}$15. Rafael Tonicelli, Anderson C. A. Nascimento, Rafael Dowsley, Jörn MüllerQuade, Hideki Imai, Goichiro Hanaoka, and Akira Otsuka. Informationtheoretically secure oblivious polynomial evaluation in the commodity-based model. Int. J. Inf. Sec., 14(1):73-84, 2015.

\section{A Our Constructions in the Big Picture}

In this section we present a graphical overview of how our constructions fits in the bigger picture of performing secure 2-party computation.

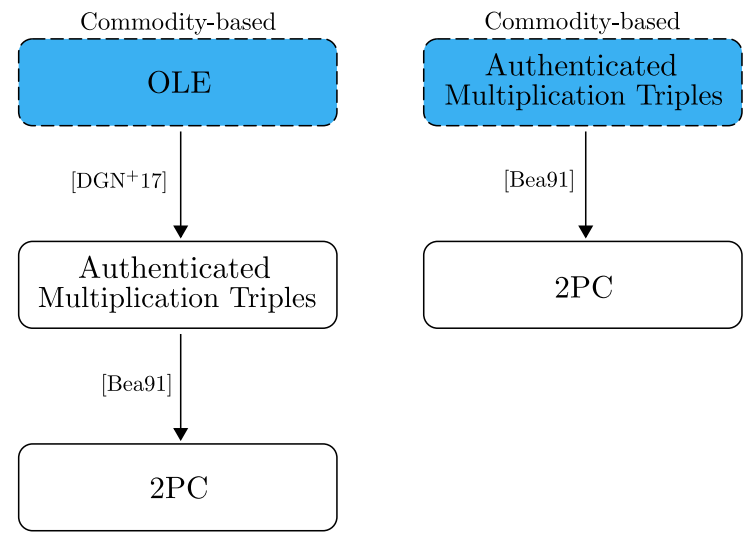

Fig. 1. An overview of how our constructions (the blue boxes with dashed lines) fits in the bigger picture of performing secure 2-party computation.

Construction 1: Commodity-based OLEs. We construct commodity-based OLEs in the commitment-hybrid model with active, statistical security and in the standard model with active, information theoretic security (with slightly worse parameter). In this construction we allow a corrupt client to collude with a minority of the servers. Using the result from $\left[\mathrm{DGN}^{+} 17\right.$, we can use the commodity-based OLEs to construct authenticated multiplication triples, which are complete for multiparty computation.

Construction 2: Commodity-based Multiplication Triples. We construct commodity-based authenticated multiplication triples in the standard model with active, information theoretic security. This construction has a slightly weaker corruption model: we do not allow collusion between clients and servers. Thus, the adversary can corrupt either one client or a minority of the servers. 


\section{B Preliminaries}

Let $[n]$ be the set of integers $\{1, \ldots, n\}$. Denote a field of size $q$ as $\mathbb{F}_{q}$ and the set of all polynomials over such field as $\mathbb{F}_{q}[X]$. All variables and operations are over $\mathbb{F}_{q}$ unless stated otherwise. $\boldsymbol{v}$ denotes a vector with entries in $\mathbb{F}_{q}$, the entries are usually denoted $v_{1}, v_{2} \ldots$ If $\boldsymbol{u}, \boldsymbol{v}$ are vectors of the same length, say $m$, then $\boldsymbol{v} * \boldsymbol{u}$ denotes the vector containing the coordinate-wise products, $\boldsymbol{v} * \boldsymbol{u}=$ $\left(v_{1} u_{1}, v_{2} u_{2}, \ldots, v_{m} u_{m}\right)$. As a shorthand, we denote $\mathbb{F}$ to be a field of arbitrary size.

\section{B.1 Security model}

We will use the UC framework of Canetti Can01 to prove our protocols secure. Informally, we compare a real protocol $\pi$ between the parties to a setting in which the parties only talk with an ideal functionality $\mathcal{F}$, which by definition is secure. To model the information the adversary learns during the protocol execution, each ideal functionality is given a leakage port on which it leaks all the information the adversary is allowed to learn. Furthermore, to model the adversary's influence over the protocol and the corrupt players, each ideal functionality is given a influence port on which it can receive messages from the adversary. To prove the real protocol secure, we construct a simulator $\mathcal{S}$ such that no adversary controlling all malicious players can make an environment distinguish between the real protocol execution and the simulator's transcript. Intuitively, the adversary gains nothing from controlling the malicious players, that he could not have simulated himself offline. In particular we use the variant in which the environment plays the role of the adversary and will prove our protocols secure under static corruption for malicious adversaries.

\section{B.2 Commodity Model}

Commodity-based cryptography works in a client-server model, where a group of clients obtain some information (called commodities) from a set of servers. In the basic setting, the clients will send a request to a server, who will reply with a single response computed from the request, whereas other settings may extend this to multiple rounds.

Following the work of Beaver, we define a two-tiered $(c, n)$-protocol $\pi=(\mathcal{C}, \mathcal{S})$ as a collection of $c+n$ probabilistic interactive Turing machines (PTM), which are divided into two groups: the clients $\mathcal{C}$ and the servers $\mathcal{S}$. The clients must be polynomial time PTM's and are assigned a unique id $i \in[c]$, and the servers are likewise assigned a unique id $j \in[n]$. We consider the basic form of two clients $c=2$ and a variable number of servers $n$, with the servers being polynomial time PTM's. Other settings, which we shall not consider, may include a variable number of clients, computationally unbounded servers, multiple rounds between clients and servers or even allowing communication between servers. 
Definition 2 (Stateless Oblivious RPC, [Bea97]). Given a two-pass protocol between client $C_{i} \in \mathcal{C}$ and server $S_{j} \in \mathcal{S}$, where the $C_{i}$ sends a request $q_{i, j}$ to the $S_{j}$, who send back the response $r_{i, j}$. This protocol is called a stateless oblivious remote procedure call (RPC) if $q_{i, j}$ is independent of $C_{i}$ 's input $x_{i}$ (apart from the length) and of any previous communications with $S_{j}$ or any other servers (apart from including tags for identifying and authenticating $C_{i}$ and $S_{j}$ ).

Definition 3 (Commodity-based Protocol, [Bea97]). A two-tiered protocol $\pi$ is commodity-based if

1. No communication between servers is necessary.

2. Servers do not need to know the identities, numbers of, or existence of other servers.

3. For each client $C_{i} \in \mathcal{C}$ and server $S_{j} \in \mathcal{S}, C_{i}$ interacts with $S_{j}$ only through stateless oblivious $R P C$ 's.

\section{B.3 Commitments}

Some of our protocol make use of a UC-secure commitment scheme, which is modelled by an ideal functionality $\mathcal{F}_{\text {Соммтт }}$. Committing to a value $x$ is denoted $\operatorname{COMMit}(x)$ and means that the committer sends $x$ to the commit functionality which notifes the other party that the commitment has been made. Opening is denoted $\operatorname{OPEN}(x)$ and means the committer sends an open command to the functionality which then sends $x$ to the other party. In a practical implementation, $\mathcal{F}_{\text {Сомміт }}$ can be implemented using a random oracle (it is well known, and trivial to prove, that applying a random oracle to the string to commit to, concatenated by random coins, gives a UC-secure commitment scheme).

\section{B.4 Oblivious Linear Evaluation}

An oblivious linear evaluation (OLE) over the finite field $\mathbb{F}$ is a primitive in which Alice inputs $a, b \in \mathbb{F}$ and Bob inputs $x \in \mathbb{F}$. Alice learns nothing and Bob learns $y=a x+b$. This primitive can be seen as a natural generalization of Oblivious Transfer (OT) Rab05 for the case $\mathbb{F}=\mathbb{F}_{2}$ or as a special case of Oblivious Polynomial Evaluation (OPE) [NP99] for the case of degree 1 polynomials. The ideal UC-functionality $\mathcal{F}_{\mathrm{OLE}}^{m}$ is defined in Figure 2, It implements $m$ OLEs in parallel.

A variant called random oblivious linear evaluation (ROLE) is a similar primitive, but where Alice receives random values $u, w_{1} \stackrel{\$}{\leftarrow} \mathbb{F}$ and Bob $v, w_{2} \stackrel{\$}{\leftarrow} \mathbb{F}$ such that $u v=w_{1}+w_{2}$. The ideal UC-functionality $\mathcal{F}_{\mathrm{ROLE}}^{m}$ is defined in Figure 3 . We show that a random oblivious linear evaluation (ROLE) is equivalent to an oblivious linear evaluation (OLE) in the same way oblivious transfer is shown to be equal to a random oblivious transfer. We define a protocol $\pi_{\mathrm{OLE}}^{m}$ that realizes $\mathcal{F}_{\mathrm{OLE}}^{m}$ with access to $\mathcal{F}_{\mathrm{ROLE}}^{m}$. This protocol (which is folklore) is formally given in Figure 4 and can be proven secure as stated in the following: 
Functionality $\mathcal{F}_{\mathrm{OLE}}^{m}$

1. Upon receiving message (inputA, $a, b$ ) from Alice with $\boldsymbol{a}, \boldsymbol{b} \in \mathbb{F}^{m}$ : if there already is a stored tuple from Alice, then ignore the message. Otherwise, store $\boldsymbol{a}$ and $\boldsymbol{b}$ and send message (inputA) on leakage port.

2. Upon receiving message (inputB, $\boldsymbol{x}$ ) from Bob with $\boldsymbol{x} \in \mathbb{F}^{m}$ : if there already is a stored tuple from Bob, then ignore the message. Otherwise, store $\boldsymbol{x}$ and send message (inputB) on leakage port.

3. Upon receiving message (deliver, A) on influence port: if $\boldsymbol{a}, \boldsymbol{b}$ and $\boldsymbol{x}$ have been stored, then send (delivered) to Alice. Otherwise, ignore the message.

4. Upon receiving message (deliver, B) on influence port: if $\boldsymbol{a}, \boldsymbol{b}$ and $\boldsymbol{x}$ have been stored, then set $\boldsymbol{y}=\boldsymbol{a} * \boldsymbol{x}+\boldsymbol{b}$ and send (output, $\boldsymbol{y}$ ) to Bob. Otherwise, ignore the message.

Fig. 2. Ideal functionality $\mathcal{F}_{\mathrm{OLE}}^{m}$ for Oblivious Linear Evaluation (OLE).

Functionality $\mathcal{F}_{\text {ROLE }}^{m}$

1. Upon receiving message (init) from both Alice and Bob, store init = true and send message (init) on leakage port.

2. Upon receiving message (corrupt, $A,\left(\boldsymbol{u}, \boldsymbol{w}_{1}\right)$ ) on influence port with $\boldsymbol{u}, \boldsymbol{w}_{1} \in$ $\mathbb{F}^{m}$ : if no values for $\boldsymbol{u}, \boldsymbol{w}_{1}$ have been stored, draw and store uniformly random $\boldsymbol{v} \in \mathbb{F}^{m}$ and compute and store $\boldsymbol{w}_{2}:=\boldsymbol{u} * \boldsymbol{v}-\boldsymbol{w}_{1}$.

3. Upon receiving message (corrupt, $\mathrm{B},\left(\boldsymbol{v}, \boldsymbol{w}_{2}\right)$ ) on influence port with $\boldsymbol{v}, \boldsymbol{w}_{2} \in$ $\mathbb{F}^{m}$ : if no values for $\boldsymbol{v}, \boldsymbol{w}_{2}$ have been stored, draw and store uniformly random $\boldsymbol{u} \in \mathbb{F}^{m}$ and compute and store $\boldsymbol{w}_{1}:=\boldsymbol{u} * \boldsymbol{v}-\boldsymbol{w}_{2}$.

4. Upon receiving message (deliver, A) on influence port, if init = true: if no values for $\boldsymbol{u}, \boldsymbol{v}, \boldsymbol{w}_{1}$ and $\boldsymbol{w}_{2}$ have been stored, draw and store uniformly random $\boldsymbol{u}, \boldsymbol{v}, \boldsymbol{w}_{1} \in \mathbb{F}^{m}$ and compute and store $\boldsymbol{w}_{2}:=\boldsymbol{u} * \boldsymbol{v}-\boldsymbol{w}_{1}$. Send (output, $\left(\boldsymbol{u}, \boldsymbol{w}_{1}\right)$ ) to Alice.

5. Upon receiving message (deliver,B) on influence port, if init $=$ true: if no values for $\boldsymbol{u}, \boldsymbol{v}, \boldsymbol{w}_{1}$ and $\boldsymbol{w}_{2}$ have been stored, draw and store uniformly random $\boldsymbol{u}, \boldsymbol{v}, \boldsymbol{w}_{1} \in \mathbb{F}^{m}$ and compute and store $\boldsymbol{w}_{2}:=\boldsymbol{u} * \boldsymbol{v}-\boldsymbol{w}_{1}$. Send (output, $\left(\boldsymbol{v}, \boldsymbol{w}_{2}\right)$ ) to Bob.

Fig. 3. Ideal functionality $\mathcal{F}_{\mathrm{ROLE}}^{m}$ for Random Oblivious Linear Evaluation (ROLE). It chooses random outputs for the parties, but lets a corrupt party choose his own outputs.

Lemma 3. The protocol $\pi_{\mathrm{OLE}}^{m} U C$-realizes $\mathcal{F}_{\mathrm{OLE}}^{m}$ in the $\mathcal{F}_{\mathrm{ROLE}}^{m}$-hybrid model.

Correctness of the protocol can be trivially checked. Security can be proven similarly to other protocols in the correlated randomness model $\left[\mathrm{IMM}^{+} 13\right.$ ]: if Bob is corrupted, the simulator emulates the ROLE by picking random $\boldsymbol{v}, \boldsymbol{w}_{2}$, extracts $\boldsymbol{x}$ from $\boldsymbol{e}$ and $\boldsymbol{v}$ and feeds it to the ideal functionality to receive $\boldsymbol{y}$. Finally the simulator chooses a random $\boldsymbol{s}$ and computes $\boldsymbol{d}=\boldsymbol{v}^{-1} *\left(\boldsymbol{y}-\boldsymbol{w}_{2}-\boldsymbol{s}\right)$, thus producing a view which is distributed identically as in the real world. Here, $\boldsymbol{v}^{-1}$ 
means the vector with entries $v_{1}^{-1}, v_{2}^{-1}, \ldots$ In the case where Alice is corrupted, the simulator emulates the ROLE by picking random $\boldsymbol{u}, \boldsymbol{w}_{1}$ and sends a random $\boldsymbol{e}$. Then, upon receiving $\boldsymbol{d}$ and $\boldsymbol{s}$ the simulator extracts $\boldsymbol{a}=\boldsymbol{d}+\boldsymbol{u}$ and $\boldsymbol{b}=$ $\boldsymbol{s}-\boldsymbol{w}_{1}-\boldsymbol{a} * \boldsymbol{e}$ and feeds them to the ideal functionality.

\[ \text { Protocol } \pi_{\mathrm{OLE}}^{m} \]
Input: Alice inputs $\boldsymbol{a}, \boldsymbol{b} \in \mathbb{F}^{m}$ and Bob inputs $\boldsymbol{x} \in \mathbb{F}^{m}$
Output: Bob outputs $\boldsymbol{y}$ such that $\boldsymbol{y}=\boldsymbol{a} * \boldsymbol{x}+\boldsymbol{b}$
Protocol:
1. Both run $\mathcal{F}_{\mathrm{ROLE}}^{m}$ such that Alice gets $\boldsymbol{u}, \boldsymbol{w}_{1}$ and Bob $\boldsymbol{v}, \boldsymbol{w}_{2}$
2. Bob computes and sends $\boldsymbol{e}=\boldsymbol{x}-\boldsymbol{v} ;$
3. Alice computes and sends $\boldsymbol{d}=\boldsymbol{a}-\boldsymbol{u}$ and $\boldsymbol{s}=\boldsymbol{w}_{1}+\boldsymbol{a} * \boldsymbol{e}+\boldsymbol{b}$
4. Bob returns $\boldsymbol{y}=\boldsymbol{w}_{2}+\boldsymbol{x} * \boldsymbol{d}-\boldsymbol{d} * \boldsymbol{e}+\boldsymbol{s}$

Fig. 4. Protocol for OLE in the $\mathcal{F}_{\mathrm{ROLE}}^{m}$-hybrid model.

\section{More Details on Commodity-based OLE}

Concrete Efficiency. We remark on the overall communication of the OLE protocol: For a single OLE instance, the oblivious $R P C$ 's consist of one tuple $\mathbb{F}^{2}$ sent from each server to each client totaling $4 n \log _{2}|\mathbb{F}|$ bits. In the computation phase, both parties send to each other one field element for each evaluation point from $d+2, \ldots, n$, which totals $2(n-(t+m+2)+1)$ field elements as $d=t+m$. In the check phase, each party sends 3 field elements to the other, and in addition we send a commitment (and its decommitting information). Let $\kappa$ be the security parameter (e.g., if SHA256 is used for the commitment $\kappa=256$ ). Then, the overall communication complexity (including the communication from the servers to the clients, and the communication between the two clients) for generating $m$ OLEs using $n$ servers tolerating up to $t$ corruptions boils down to

$$
2(3 n-t-m+2) \log _{2}|\mathbb{F}|+2 \kappa .
$$

Doing Without Commitments. The only reason we use a commitment is that if Bob would immediately send $B\left(t_{A}\right)$ to Alice, the proof of Lemma 2 would break down because Alice would now know $t+1$ and not $t$ points on $B(x)$, at the time where she has to answer Bob's challenge. Therefore, in the view of Alice, there is only 1 degree of freedom for $B(x)$ instead of 2 , as we need in the proof. We can even show that there is an attack on the protocol in this case.

However, this is easy to fix, we just set the degree of polynomials $A(x), B(x)$ to be $d=t+m+1$ instead of $t+m$, and change the protocol such that in the Check Phase, Bob sends $B\left(t_{A}\right), C_{2}\left(t_{A}\right)$ in the clear along with $t_{B}$. Alice can now do her check immediately and return her answer $A\left(t_{B}\right), C_{1}\left(t_{B}\right)$ to Bob. We can 
prove the equivalent of Lemma 2 for the modified protocol using the same proof: even if Alice now learns $B\left(t_{A}\right)$ before she has to answer she is still at least 2 points short of being able to determine $B(x)$, and this is the crucial property that makes the proof go through. The simulation for the modified protocol and the proof that it works is the same as before. The price we pay for this is that we need $n=2 d+1=2 t+2 m+3$, so we need 2 servers more than before. In summary, we have

Corollary 1. Assume that $n=2 t+2 m+3$ and that $|\mathbb{F}|$ is exponential in the security parameter. Then protocol $\pi_{\mathrm{ROLE}}^{m}$ is an implementation of $\mathcal{F}_{\mathrm{ROLE}}^{m}$ with statistical UC-security. The protocol tolerates a static adversary corrupting one client and at most $t \leq \frac{n-2 m-3}{2}$ servers. The simulation is perfect unless an error event occurs, which has probability at most $\frac{n}{|\mathbb{F}|-(n+m)}$.

Smaller Fields. The protocol can be modified to work even with small fields where $|\mathbb{F}|$ is not exponentially large in the security parameter: the argument that the check phase makes a mistake with probability a most $n /(|\mathbb{F}|-(m+n))$ holds for any field. In particular, we will get at most constant error probability $p$ as long as $|\mathbb{F}|>n / p+m+n$. Then we can get negligible error probability if we repeat the check phase $\kappa$ times where $\kappa$ is the security parameter. This will give error probability at most $p^{\kappa}$.

\section{Allowing Servers to be Memoryless}

We now look at practical aspect of the commodity model. As mentioned in the introduction, it will clearly be an advantage if the servers in our model do not need memory in the sense that they do not have to remember who they talked to or what was sent. This would mean that a server will not have to administrate identities and log-in credentials, and could simply generate the required data on the fly, say, against payment in some cryptocurrency. An obvious problem with this goal is that the data sent to two clients Alice and Bob must be correlated and a memoryless server cannot make this happen on its own. We now informally sketch a solution to this: We will assume that clients can communicate with servers such that clients can authenticate the identity of servers, but not necessarily the other way around (in practice, one may think of 1-way TLS here.)

We also assume that Alice and Bob interact before talking to the servers indeed this is necessary to create any correlation if the servers have no memory. We assume a 2-way authenticated channel for this, indeed this seems necessary if there are several clients, otherwise an honest Alice could not know with whom she is doing secure computation. Alice and Bob would then agree on a common nonce $n_{A B}$, as well as a parameter par specifying what they will request from the server, as well as the identity $i d$ of the server. For the case of our protocol, we would have par $=(\mathbb{F}, s, i d)$ where $\mathbb{F}$ is the field to use for the OLEs, $s$ is the number of OLEs to request and $i d$ is the server identifier.

In a naive solution, Alice would send ("A", par, $n_{A B}$ ) to the server who will use this and a private PRF key $K$ to generate Alice's data, and something similar 
is done for Bob. However, this is clearly not secure: if Alice is corrupt, she can send both ("A", par, $n_{A B}$ ) and ("B", par, $n_{A B}$ ) to each honest server, get Bob's data and break the protocol.

We solve this by generating the nonce such that Alice and Bob each know different secrets relating to the nonce and hence cannot impersonate the other party towards the server. In the simplest case where a nonce is used for only one server, a straightforward way to do this is to make use of a one-way function $f:\{0,1\}^{k} \mapsto\{0,1\}^{k}$ where $k$ is a security parameter. Then Alice chooses $x_{A} \in$ $\{0,1\}^{k}$ at random, similarly Bob chooses $x_{B}$ and we let $n_{A, B}=f\left(x_{A}\right) \| f\left(x_{B}\right)$ where $\|$ denotes concatenation.

Now, party $P \in\{A, B\}$ would send ("P", par, $x_{P}, n_{A B}$ ). The server checks that $x_{P}$ is correct with respect to $n_{A B}$ and only then will it send data to $P$. In this case we can instantiate $f$ efficiently using a hash function, for instance.

For the security of this solution, note that we just need to make sure that the data sent from an honest server to an honest client is secure, since all other data is known and can be modified by the adversary anyway. So assume Alice is honest and agrees on nonce $n_{A B}$ and par with corrupt Bob, and let $d_{A}$ be the data that honest server $S$ will returns to Alice. Now, if Bob sends any request to $S$ that contains something different from par, $n_{A B}$ then $S$ will return something that is (pseudo)uncorrelated to $d_{A}$. If the request does contain par, $n_{A B}$, then $S$ may return either nothing or the data Bob is allowed to get, which is fine. It will only return $d_{A}$ if the request contains $x_{A}$, and this happens only with negligible probability since $f$ is one-way.

It is also possible to use one nonce for all servers. In that case we cannot let Alice simply reveal a preimage to the server. If the server is corrupt it can send $x_{A}$ to Bob who can then do the same attack as before on honest servers. Instead we can let Alice generate a public key $v k_{A}$ for a secure signature scheme, while Bob generates $v k_{B}$. Now, the request sent by Alice will be of form ("A", par, $\sigma_{A}, n_{A B}$ ), where $\sigma_{A}$ is a signature on $n_{A B}$ and par, and where $p a r=\left(\mathbb{F}, s, i d, v k_{A}, v k_{B}\right)$ The server only sends back data if the signature verifies under the public key found in par, and if its own name occurs in par. Note that this last checks prevents a corrupt server from replaying a request to an honest server, and hence security can be argued in a similar way as before.

We remark that a practical implementation of any information theoretically secure MPC needs to implement the secure channels using encryption and (usually) a PKI, which is only computationally secure. We are in a similar situation, only we consider also the authentication aspect: if we assumed ideal authentic channels, the servers could generate data based on the ID's of the parties they know they are talking to. If we do not assume this, we have to use computational assumptions. 